\title{
Objective quantification of contrast enhancement of unruptured intracranial aneurysms: a high-resolution vessel wall imaging validation study
}

\author{
Jorge A. Roa, MD, ${ }^{1,2}$ Mario Zanaty, MD, ${ }^{2}$ Carlos Osorno-Cruz, BS, ${ }^{2}$ Daizo Ishii, MD, ${ }^{2}$ \\ Girish Bathla, MD, ${ }^{3}$ Santiago Ortega-Gutierrez, MD, MSc, ${ }^{1-3}$ David M. Hasan, MD, ${ }^{2}$ and \\ Edgar A. Samaniego, MD, MS ${ }^{1-3}$
}

Departments of ${ }^{1}$ Neurology, ${ }^{2}$ Neurosurgery, and ${ }^{3}$ Radiology, University of lowa Hospitals and Clinics, lowa City, lowa

\begin{abstract}
OBJECTIVE High-resolution vessel wall imaging (HR-VWI) has emerged as a valuable tool in assessing unruptured intracranial aneurysms (UIAs). There is no standardized method to quantify contrast enhancement of the aneurysm wall. Contrast enhancement can be objectively measured as signal intensity (SI) or subjectively adjudicated. In this study, the authors compared the different methods to quantify wall enhancement of UIAs and determined the sensitivity and specificity of each method as a surrogate of aneurysm instability. They also compared SI quantification between scanners from different manufacturers.
\end{abstract}

METHODS The University of lowa HR-VWI Project database was analyzed. This database compiles patients with UIAs who prospectively underwent HR-VWI using a 3T MRI scanner. The mean and maximal SI values of the aneurysm wall, pituitary stalk, and genu of the corpus callosum were used to compare 3 different measurement methods: 1) aneurysm enhancement ratio $\left.\mathrm{AER}=\left(\left.\mathrm{S}\right|_{\text {wall post }}-\left.\mathrm{SI}\right|_{\text {wall pre }}\right) /\left.\mathrm{S}\right|_{\text {wall pre }} ; 2\right)$ aneurysm-to-pituitary stalk contrast ratio $\mathrm{CR}_{\text {stalk }}=\mathrm{SI}_{\text {wall post }} / S I_{\text {stalk }}$ posti and 3$)$ aneurysm enhancement index AEI = ([SI wall post $\left.\left./\left.S\right|_{\text {brain post }}\right]-\left[\left.S\right|_{\text {wall pre }} / S I_{\text {brain pre }}\right]\right) /\left(\left.S\right|_{\text {wall pre }} /\left.S\right|_{\text {brain pre }}\right)($ where "pre" indicates precontrast images and "post" indicates postcontrast images). Size $\geq 7 \mathrm{~mm}$ was used as a surrogate of aneurysm instability for receiver operating characteristic (ROC) curve analysis. To determine if the objective quantification of SI varies among scanners from different manufacturers, 9 UIAs underwent the same HR-VWI protocol using a 3T General Electric (GE) scanner and a 3T Siemens scanner. Three UIAs also underwent a third scanning procedure on a unit with a different magnet strength (7T GE).

RESULTS Eighty patients with 102 UIAs were included in the study. The mean age was $64.5 \pm 12.2$ years, and 64 $(80 \%)$ patients were women. UIAs $\geq 7 \mathrm{~mm}$ had significantly higher Sls than smaller UIAs $(<7 \mathrm{~mm})$ : AER $=0.82$ vs 0.49 , $p<0.001 ; \mathrm{CR}_{\text {stalk }}=0.84$ vs $0.61, p<0.001$; and $\mathrm{AEI}=0.81$ vs $0.48, p<0.001$. ROC curves demonstrated optimal sensitivity of $81.5 \%$ for $\mathrm{CR}_{\text {stalk }} \geq 0.60,75.9 \%$ for $\mathrm{AEI} \geq 0.50$, and $74.1 \%$ for $\mathrm{AER} \geq 0.49$. Intermanufacturer correlation between $3 \mathrm{~T}$ GE and $3 \mathrm{~T}$ Siemens MRI scanners for $\mathrm{CR}_{\text {stak }}$ using mean and maximal SI values was excellent (Pearson coefficients $>0.80, p<0.001)$. A similar correlation was identified among the $3 \mathrm{UIAs}$ that underwent $7 \mathrm{~T}$ imaging.

CONCLUSIONS $\mathrm{CR}_{\text {stak }}$ using maximal SI values was the most reliable objective method to quantify enhancement of UIAs on HR-VWI. The same ratios were obtained between different manufacturers and on scans obtained using magnets of different strengths.

https://thejns.org/doi/abs/10.3171/2019.12.JNS192746

KEYWORDS high-resolution vessel wall imaging; magnetic resonance imaging; aneurysm; circumferential enhancement; validation; aneurysmal subarachnoid hemorrhage; vascular disorders

$\mathrm{U}$ NRUPTURED intracranial aneurysms (UIAs) pose a therapeutic dilemma, as the risk-benefit of therapeutic interventions has to be balanced against the natural history of the disease. Early recognition of brain aneurysms with a high risk of rupture is key when deciding treatment. Unfortunately, there is no biomarker of aneurysm instability that has been prospectively validated. High-resolution vessel wall imaging (HR-VWI) has

ABBREVIATIONS $A C O A=$ anterior communicating artery; $A E I=$ aneurysm enhancement index; $A E R=$ aneurysm enhancement ratio; $A U C=$ area under the curve; $B A=$ basilar artery; $\mathrm{CAWE}=$ circumferential aneurysm wall enhancement; $\mathrm{CE}-\mathrm{MRA}=$ contrast-enhanced $\mathrm{MR}$ angiography; $\mathrm{CR}_{\text {stalk }}=$ aneurysm-to-pituitary stalk contrast ratio; HR-VWI = high-resolution vessel wall imaging; ISUIA = International Study of Unruptured Intracranial Aneurysms; PCOA = posterior communicating artery; ROC = receiver operating characteristic; $\mathrm{ROI}=$ region of interest; $\mathrm{SI}=$ signal intensity; $\mathrm{UIA}=$ unruptured intracranial aneurysm.

SUBMITTED October 10, 2019. ACCEPTED December 5, 2019.

INCLUDE WHEN CITING Published online February 7, 2020; DOI: 10.3171/2019.12.JNS192746. 
TABLE 1. Ratios and indexes used to objectively quantify aneurysm wall enhancement

\begin{tabular}{|c|c|c|}
\hline Method & Formula* & Authors \& Year \\
\hline AER & $\left(S I_{\text {wall post }}-S I_{\text {wall pre }}\right) / S I_{\text {wall pre }}$ & Wang et al., 2018 \\
\hline AEl & $\left(\left[\left.S\right|_{\text {wall post }} / S I_{\text {brain post }}\right]-\left[\left.S\right|_{\text {wall pre }} / S I_{\text {brain pre }}\right]\right) /\left(S I_{\text {wall pre }} / S I_{\text {brain pre }}\right)$ & Omodaka et al., 2016 \\
\hline $\mathrm{CR}_{\text {stalk }}$ & $\mathrm{SI}_{\text {wall post }} / \mathrm{SI}$ stalk post & Omodaka et al., 2019 \\
\hline
\end{tabular}

* Each formula was computed using the mean and maximal $\mathrm{SI}_{\text {wall. }}$.

emerged as a valuable tool in assessing unstable UIAs. ${ }^{3}$ There are promising observations in the characterization of aneurysm wall enhancement as a biomarker of aneurysm wall inflammation, growth, and rupture. ${ }^{19}$

However, there is significant heterogeneity among the imaging protocols used to characterize UIAs with HRVWI. ${ }^{28}$ Furthermore, there is no consensus on the standard definition of wall enhancement. ${ }^{24}$ Most studies have classified wall enhancement subjectively into strong/avid versus faint/no enhancement, or focal versus circumferential wall enhancement, based on the assessment by 2 or more adjudicators. Other studies have used objective data generated from the quantification of signal intensity (SI) in the aneurysm wall to define enhancement. Several formulas have been used to standardize enhancement measurements, including ratios generated through comparisons with the pituitary stalk ${ }^{15,16}$ and those considering SI measurements in pre- versus postcontrast sequences. ${ }^{26,27}$

We aimed to compare the different methods of aneurysm wall enhancement measurement to determine the most sensitive and specific measurements. Moreover, our goal was to validate these measurements between scanners from different manufacturers and magnet strengths.

\section{Methods}

\section{Patient Population and Data Collection}

After obtaining IRB approval, we analyzed the University of Iowa HR-VWI Project database. This database is prospectively acquired and includes patients with UIAs from January 2015 to August 2019. At our institution, every patient with a UIA undergoes 3T HR-VWI. The IRB was amended to obtain an additional image in a subset of patients using a scanner from a different manufacturer and sometimes a third scan using a scanner with a different magnet strength (7T). Demographics and clinical information, including age, sex, and comorbidities, were obtained from electronic medical records.

\section{Imaging Acquisition}

Images are routinely acquired using a 3T Siemens MRI scanner (MAGNETOM Skyra, Siemens). The HR-VWI protocol included a 3D T1-weighted SPACE FSE (3D sampling perfection with application-optimized contrasts using different flip-angle evolutions fast spin echo) and a 3D T2-weighted sequence. Five minutes after an intravenous injection of $0.1 \mathrm{mmol} / \mathrm{kg}$ gadolinium-based contrast agent (Gadavist, Bayer Pharmaceuticals), a postcontrast 3D T1-weighted SPACE FSE sequence was obtained, and contrast-enhanced MR angiography (CE-MRA) was per- formed. The reproducibility of results was tested on a $3 \mathrm{~T}$ GE MRI scanner (GE Healthcare) in 9 UIAs that underwent additional HR-VWI. Three UIAs underwent a third scan on a 7T GE MRI scanner (GE Healthcare). Technical parameters used for imaging acquisition on each scanner are described in Supplemental Tables 1-3.

\section{HR-VWI Assessment}

All images were analyzed with the Picture Archiving Communication System (PACS, version 12.1.6.1005, Carestream Vue). Aneurysm size (diameter and neck) was measured on CE-MRA images. After 6-fold magnification and autocorrection of viewer windowing, the aneurysm was manually coregistered on both pre- and postcontrast T1-weighted sequences in all 3 planes (axial, coronal, and sagittal). A 2D region of interest (ROI) of the aneurysm wall was drawn at the level of the maximal aneurysm diameter. A combination of CE-MRA and 3D T1-weighted SPACE (precontrast) images were used as a reference to exclude the aneurysm lumen and delineate the inner surface of the aneurysm wall, while both 3D T2-weighted sequences and 3D T1-weighted SPACE (postcontrast) were collectively used to distinguish artifacts such as cerebrospinal fluid, meninges, and veins. ROIs were visually and statistically analyzed to determine that they encompassed the same aneurysm wall area on pre- and postcontrast T1weighted images (Fig. 1).

Three different methods of the aneurysm wall enhancement measurement were compared: the aneurysm enhancement ratio (AER), the aneurysm enhancement index (AEI), and the aneurysm-to-pituitary stalk contrast ratio $\left(\mathrm{CR}_{\text {stalk }}\right)$. Table 1 summarizes the different formulas and SI measurements used for each method.

As proposed by Wang et al., ${ }^{27}$ SI values measured in the aneurysm wall from each projection $\left(\mathrm{SI}_{\text {wall }}\right)$ on pre- and postcontrast $\mathrm{T} 1$-weighted images were used to calculate the AER as follows: $\left(\mathrm{SI}_{\text {wall post }}-\mathrm{SI}_{\text {wall pre }}\right) / \mathrm{SI}_{\text {wall pre }}$. Then, following a similar method described by Omodaka et al., ${ }^{16}$ SI was quantified in coregistered ROIs of $20 \mathrm{~mm}^{2}$ drawn over the genu of the corpus callosum in all views $\left(\mathrm{SI}_{\text {brain }}\right)$. The AEI was calculated adjusting $\mathrm{SI}_{\text {wall }}$-to-SI $\mathrm{SI}_{\text {brain }}$ measurements as follows: ([SI $\left.\left.\mathrm{SI}_{\text {wall post }} / \mathrm{SI}_{\text {brain post }}\right]-\left[\mathrm{SI}_{\text {wall pre }} / \mathrm{SI}_{\text {brain pre }}\right]\right) /$ $\left(\mathrm{SI}_{\text {wall pre }} / \mathrm{SI}_{\text {brain pre }}\right)$.

The third method analyzed was the $\mathrm{CR}_{\text {stalk }}$. As described elsewhere, 4 different SI points were randomly sampled throughout the pituitary stalk on the sagittal postcontrast T1-weighted images (Fig. 2). ${ }^{15}$ The mean SI of the aneurysm wall on the postcontrast T1-weighted sequence ( $\mathrm{SI}_{\text {wall post }}$ ) was divided by the SI of all sampled points over the pituitary stalk to calculate the $\mathrm{CR}_{\text {stalk }}$ as follows: $\mathrm{SI}_{\text {wall post }} / \mathrm{SI}_{\text {stalk post }}$. 
TABLE 2. Baseline characteristics

\begin{tabular}{|c|c|c|c|}
\hline Variable & Overall* & $\begin{array}{l}\text { Enhancing } \\
\text { UIAs } \\
(n=64) \dagger\end{array}$ & $\begin{array}{c}\text { Nonenhancing } \\
\text { UIAs } \\
(n=38)\end{array}$ \\
\hline Mean age, yrs \pm SD & $64.5 \pm 12.2$ & $66.6 \pm 11.9$ & $59.9 \pm 10.3$ \\
\hline Women & $64(80)$ & $50(78.1)$ & $32(84.2)$ \\
\hline Currently smoking & $42(52.5)$ & $33(51.6)$ & $22(57.9)$ \\
\hline Hypertension & $45(56.3)$ & $36(56.3)$ & $23(60.5)$ \\
\hline Diabetes & $12(15)$ & $10(15.6)$ & $4(10.5)$ \\
\hline Hyperlipidemia & $34(42.5)$ & $25(39.1)$ & $16(42.1)$ \\
\hline Family history of IAs & $7(8.75)$ & $5(7.8)$ & $5(13.2)$ \\
\hline $\begin{array}{l}\text { Mean aneurysm size, } \\
\mathrm{mm} \text { (range) }\end{array}$ & $8.7(3-31)$ & $10.8(3-31)$ & $5.1(3-11)$ \\
\hline$\geq 7$ & $54(52.9)$ & $44(68.8)$ & $10(26.3)$ \\
\hline$<7$ & 48 (47.1) & $20(31.3)$ & $28(73.7)$ \\
\hline \multicolumn{4}{|l|}{ Aneurysm location } \\
\hline ICA & $25(24.5)$ & $22(34.4)$ & $3(7.9)$ \\
\hline $\mathrm{ACOA}$ & $16(15.7)$ & $7(10.9)$ & $9(23.7)$ \\
\hline ACA & $5(4.9)$ & $3(4.7)$ & $2(5.3)$ \\
\hline MCA & $27(26.5)$ & $14(26.5)$ & $13(34.2)$ \\
\hline PCoA & $5(4.9)$ & $3(4.7)$ & $2(5.3)$ \\
\hline BA & $19(18.6)$ & $12(18.8)$ & $7(18.4)$ \\
\hline SCA & $3(2.9)$ & $1(1.6)$ & $2(5.3)$ \\
\hline VA & $2(2.0)$ & $2(3.1)$ & $0(0)$ \\
\hline \multicolumn{4}{|c|}{$\begin{array}{l}\text { ACA = anterior cerebral artery; } I A=\text { intracranial aneurysm; ICA = internal } \\
\text { carotid artery; } M C A=\text { middle cerebral artery; } S C A=\text { superior cerebellar artery; } \\
V A=\text { vertebral artery. } \\
\text { Values are presented as the number (\%) unless otherwise indicated. } \\
\text { *In overall counts, frequencies for demographics are calculated over total } \\
\text { number of patients }(n=80) \text {, whereas size and location are presented over total } \\
\text { number of UIAs ( } n=102) \text {. } \\
\dagger \text { According to } C R_{\text {stalk }} \geq 0.60 \text { using maximal SI values. }\end{array}$} \\
\hline
\end{tabular}

Some UIAs show a pattern of focal enhancement, while others demonstrate uniform circumferential aneurysm wall enhancement (CAWE) on HR-VWI. To define whether the maximal SI on the aneurysm wall should be used instead of the mean SI, we calculated the AER, AEI, and $\mathrm{CR}_{\text {stalk }}$ using the maximal and mean SIs on both preand postcontrast T1-weighted images. Consequently, a total of 6 different objective approaches to measure SI were analyzed (Table 1).

\section{Statistical Analysis}

Continuous variables are presented as mean $\pm \mathrm{SD}$, and categorical variables are presented as frequency and percentage. Differences in aneurysm enhancement were statistically assessed using the Student t-test. A 2-sided p < 0.05 was considered significant. Based on results from the ISUIA (International Study of Unruptured Intracranial Aneurysms $)^{30}$ and other observational studies, UIAs $\geq 7$ $\mathrm{mm}$ located in the anterior communicating artery (ACoA), posterior communicating artery (PCoA), and basilar artery (BA) are more likely to rupture and were categorized as unstable. We used these variables (size and location) to perform area under the curve receiver operating characteristic (AUC-ROC) analysis., ${ }^{2,12}$ All statistical analyses were performed using IBM SPSS Statistics (version 25.0, IBM Corp.).

\section{Results}

A total of 80 patients with 102 UIAs were included. The mean age was $64.5 \pm 12.2$ years, and $64(80 \%)$ patients were women (Table 2). Most aneurysms had saccular morphology (96 aneurysms, 94.1\%). Correlation statistics demonstrated high agreement for areas covered by ROIs in the axial, coronal, and sagittal projections for both pre- and postcontrast T1-weighted images (Pearson coefficients $>0.92, \mathrm{p}<0.001$ ).

The Student t-test showed that aneurysms $\geq 7 \mathrm{~mm}$ had significantly higher maximal SI measurements for AER (0.82 vs $0.49, \mathrm{p}<0.001), \mathrm{CR}_{\text {stalk }}(0.84$ vs $0.61, \mathrm{p}<0.001$ ), and AEI $(0.81$ vs $0.48, \mathrm{p}<0.001)$ than did smaller aneurysms. An analysis of aneurysms located in the ACoA, PCoA, and BA, regardless of size, also reported increased maximal SI values for AER (0.71 vs 0.59, $\mathrm{p}=0.154$ ), $\mathrm{CR}_{\text {stalk }}(0.73$ vs $0.72, \mathrm{p}=0.955)$, and AEI (0.71 vs 0.58 , $\mathrm{p}=0.135$ ) compared with aneurysms in other locations. However, the differences for location were statistically nonsignificant.

The best AUC in subsequent ROC analyses was achieved with $\mathrm{CR}_{\text {stalk }}$ using the maximal SI (0.776), followed by AER (0.738) and AEI (0.730) (Fig. 3). Setting the specificity of all ratios to $60 \%$ or more, curve point coordinates demonstrated an optimal sensitivity of $81.5 \%$ for $\mathrm{CR}_{\text {stalk }} \geq 0.60,75.9 \%$ for $\mathrm{AEI} \geq 0.50$, and $74.1 \%$ for AER $\geq 0.49$. The cutoffs for all the ratios using maximal and mean SI are summarized in Supplemental Table 4.

Finally, similar HR-VWI protocols were used for both 3T Siemens and 3T GE MRI scanning of 9 UIAs (Table

TABLE 3. SI measurements on pre- and postcontrast T1-weighted images in 9 UIAs using different MRI units from manufacturers

\begin{tabular}{lccccc}
\hline MRI Scanner & Mean SI & Max SI & Mean SI $_{\text {post }}$ & Max SI & post $_{\text {stalk }}{ }^{*}$ \\
\hline 3T Siemens & 155.9 & 287.7 & 247.9 & 437.8 & $0.71 / 0.42$ \\
\hline 3T GE & 121.3 & 194.9 & 183.9 & 288.3 & $0.68 / 0.41$ \\
\hline 7T GE $\dagger$ & 232.2 & 402.3 & 378.8 & 671.0 & $0.79 / 0.45$ \\
\hline
\end{tabular}

Max $=$ maximal; $\mathrm{SI}_{\text {post }}=$ postcontrast $\mathrm{SI} ; \mathrm{SI}_{\text {pre }}=$ precontrast $\mathrm{SI}$.

* The $\mathrm{CR}_{\text {stalk }}$ is included for comparison: maximal $\mathrm{CR}_{\text {stalk }} /$ mean $\mathrm{CR}_{\text {stalk }}$.

† Only 3 UIAs underwent 7T GE MRI. 
CE MRA


T1 PRE


T1 POST

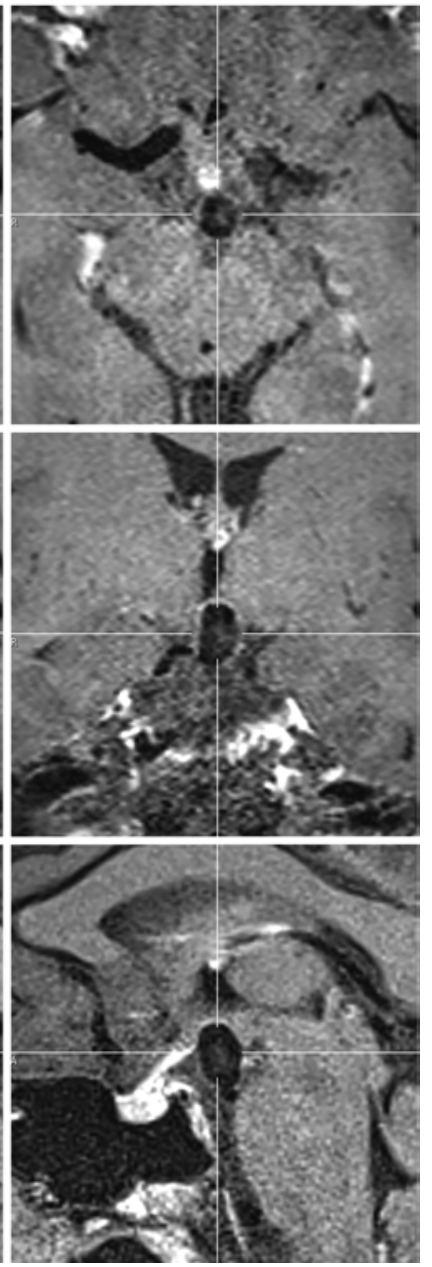

FIG. 1. Coregistration of CE-MRA and pre- and postcontrast T1-weighted sequences in a basilar tip UIA on axial (A), coronal (B), and sagittal (C) projections.

3). Absolute SI values were significantly lower on the GE scanner than on the Siemens scanner (mean $\mathrm{SI}_{\text {wall pre }}$ : 121.3 vs 155.9 ; maximal $\mathrm{SI}_{\text {wall pre }}: 194.9$ vs 287.7 ; mean $\mathrm{SI}_{\text {wall post }}$ : 183.9 vs 247.9 ; maximal $S_{\text {wall post }} 288.3$ vs $437.8, \mathrm{p}<0.001$ ). However, Pearson coefficients demonstrated an excellent correlation between the two scanners for $\mathrm{CR}_{\text {stalk }}$ using mean SI values (Pearson coefficient $=0.975, p<0.001$ ) and maximal SI values (Pearson coefficient $=0.814, p=0.008$ ). A similar correlation pattern for $\mathrm{CR}_{\text {stalk }}$ was established in the 3 UIAs that underwent 7T GE MRI (Pearson coefficients $>0.95)$. On the other hand, the intermanufacturer correlation using AER and AEI was negligible and statistically nonsignificant (Pearson coefficients $<0.40, \mathrm{p}>0.20$ ).

\section{Discussion}

Clinical and histological correlations have suggested that increased contrast enhancement of the aneurysm wall is a surrogate of aneurysm instability and increased risk of rupture. ${ }^{24,28}$ Enhancement quantification has been performed mostly subjectively by experienced adjudica- tors. ${ }^{2,12}$ Several objective methods have been described to quantify enhancement and SI. This study compared all the different objective methods and demonstrated that $\mathrm{CR}_{\text {stalk }}$ using maximal SI is the best predictor of aneurysm instability. Moreover, we demonstrated the reproducibility of this technique between scanners made by different manufacturers, which would be pivotal in a multicenter prospective clinical trial.

Inflammation of the aneurysm wall, usually initiated by a hemodynamic insult, may result in dysfunction of endothelial and vascular smooth muscle cells, local activation of cytokines, degradation of the extracellular matrix, aneurysm remodeling, and rupture. ${ }^{4}$ Several studies have correlated enhancement of the aneurysm wall on HR-VWI with inflammatory histopathological changes: increased inflammatory cells,,${ }^{8,9,22}$ myeloperoxidase activity, and vasa vasorum proliferation..$^{10,13,22,25}$ A histopathological study performed by our group demonstrated that UIAs with avid enhancement had increased macrophage infiltration and cellularity in comparison with aneurysms with a mildly enhancing or no enhancing wall. ${ }^{9}$ This sug- 


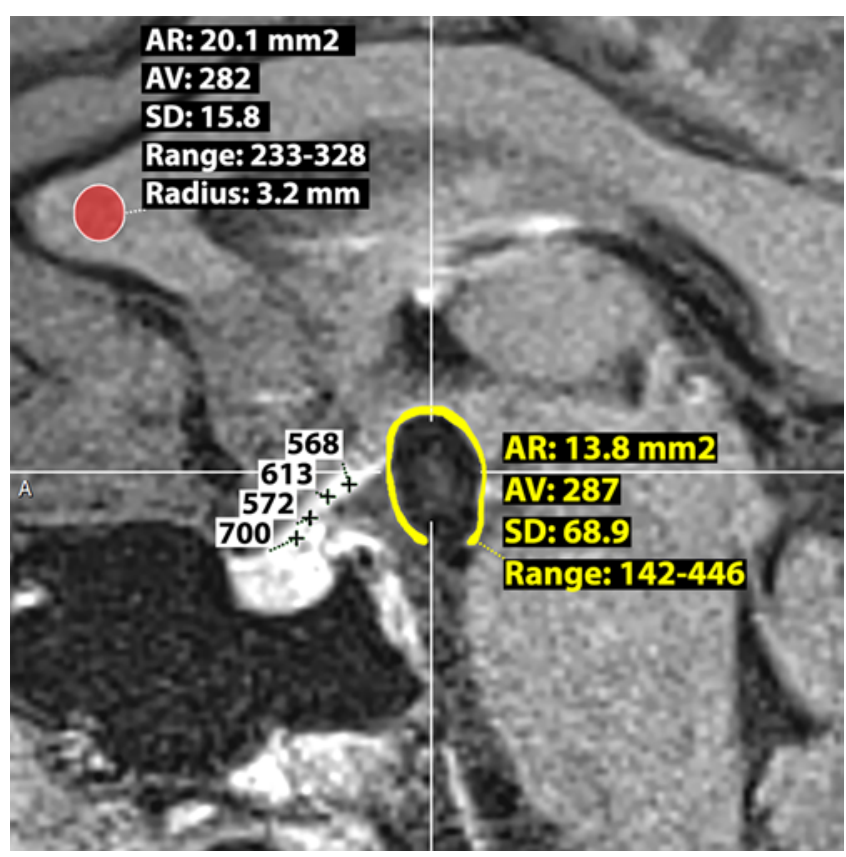

FIG. 2. Postcontrast T1-weighted sagittal projection of a basilar tip aneurysm showing the ROI of the aneurysm wall (yellow), sampling of the genu of the corpus callosum (red), and sampling of the pituitary stalk (black) for normalization. In this case, the maximal $\mathrm{CR}_{\text {stalk }}$ would be calculated as follows: $446 / 700=0.63$. AR = area; $A V=$ average. Figure is available in color online only.

gests that weakened arterial walls of unstable UIAs exhibit increased contrast enhancement on HR-VWI, and these changes might be explained by an active inflammatory/ vasculopathic reaction in the aneurysm wall. ${ }^{19}$

Edjlali et al..$^{5}$ analyzed 108 UIAs with HR-VWI and introduced the concept of CAWE as the presence of "circumferential aneurysmal wall enhancement." CAWE was more commonly seen in unstable than stable UIAs (27/31 [87\%] vs 22/77 [28.5\%], respectively; $p<0.0001$ ). Omodaka et al. proposed two different standardized tools to objectively assess wall enhancement: 1) $\mathrm{CR}_{\text {stalk }}$ on postcontrast imaging, and 2) AEI using matched volumes on pre- and postcontrast T1-weighted images in the right frontal lobe as reference. ${ }^{16}$ Later on, the same group compared $\mathrm{CR}_{\text {stalk }}$ in 69 stable UIAs, 26 evolving UIAs, and 67 ruptured aneurysms, reporting significantly higher $\mathrm{CR}_{\text {stalk }}$ values in evolving UIAs than in stable UIAs (0.54 vs 0.34 , $\mathrm{p}<0.0001$ ), but lower than those in ruptured aneurysms (0.54 vs 0.83 , $\mathrm{p}<0.0002) .{ }^{15}$ Wang et al..$^{27}$ showed significantly lower enhancement values in UIAs compared with ruptured aneurysms $(0.63$ vs $0.90, \mathrm{p}<0.001)$ using AER.

In this study, we compared all of these objective modalities for quantification of wall enhancement in UIAs. Omodaka et al. ${ }^{15}$ reported an optimal cutoff value for $\mathrm{CR}_{\text {stalk }} \geq 0.39$ to distinguish evolving from stable UIAs (AUC 0.80 ), with a sensitivity of $88 \%$ and a specificity of $63 \%$. Our analysis suggested the same cutoff value for $\mathrm{CR}_{\text {stalk }}$ using mean SI values. In a different publication, Omodaka et al. ${ }^{16}$ used maximal SI ratios to distinguish ruptured aneurysms $(n=28)$ from UIAs $(n=76)$. The au-
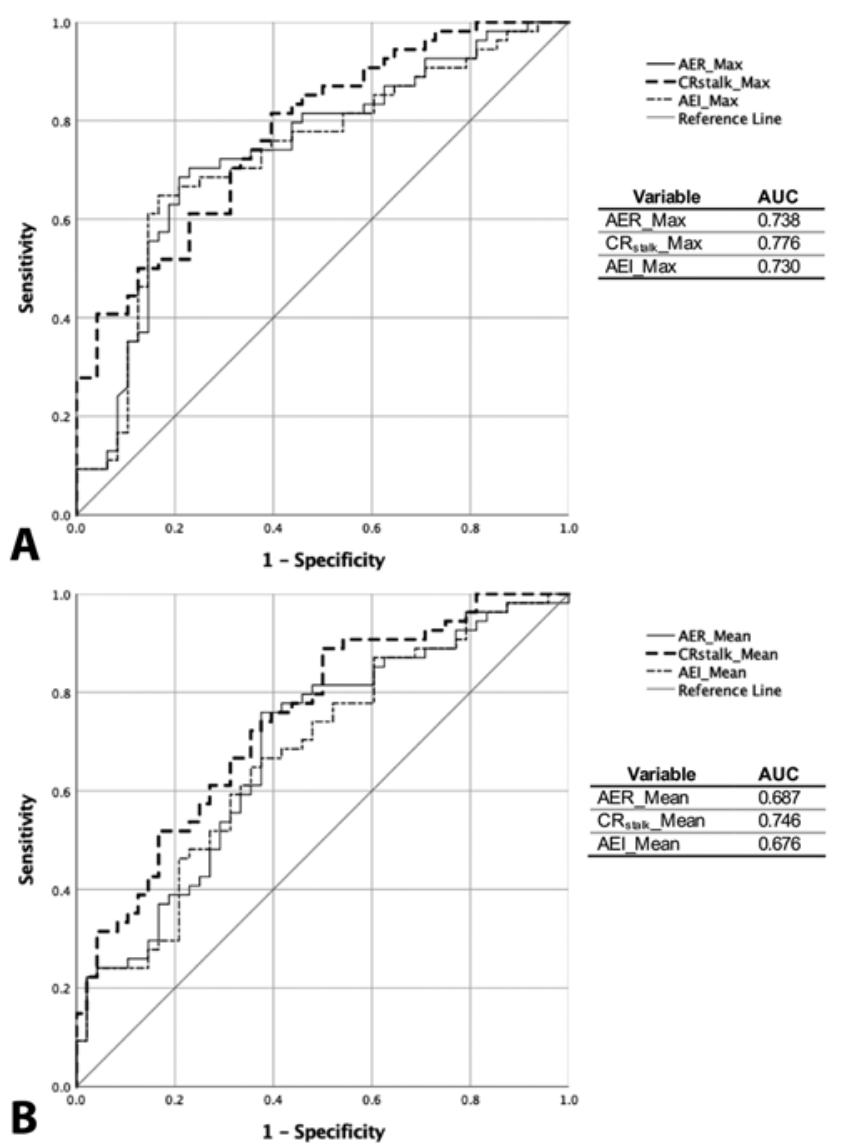

FIG. 3. ROC curves for $A E R, C_{\text {stalk }}$, and $A E I$ using maximal (Max) SI values $(\mathbf{A})$ and mean $\mathrm{SI}$ values $(\mathrm{B})$.

thors reported that $\mathrm{CR}_{\text {stalk }} \geq 0.64$ achieved a sensitivity of $75 \%$ and specificity of $83 \%$ (AUC $=0.84$ ), whereas AEI $\geq$ 0.53 achieved a sensitivity of $96 \%$ and specificity of $43 \%$ $(\mathrm{AUC}=0.75)$. We also found similar cutoffs for $\mathrm{CR}_{\text {stalk }}$ (0.60) and AEI (0.50) using maximal SI values. Using a cutoff value for AER $\geq 0.615$ to differentiate ruptured intracranial aneurysms $(n=19)$ from UIAs $(n=87)$, Wang et al. ${ }^{27}$ achieved an AUC of 0.798 , sensitivity of $89.5 \%$, and specificity of $63.2 \%$. Although we found lower cutoffs for AER $(\geq 0.49)$, our predictive measurements were lower for sensitivity (74\%) but similar for specificity (approximately $60 \%$ ). Overall, the cutoffs found in our study for each enhancement ratio/index correlate with those reported in previous studies. Minor differences in predictive measurements might be explained by the sample size, heterogeneity of the samples, and statistical power of each study.

Our study determined that the best predictor of aneurysm instability is $\mathrm{CR}_{\text {stalk }}$ using maximal $\mathrm{SI}(\geq 0.60$; sensitivity of $81.5 \%$ and specificity of $61 \%$ ). HR-VWI is a relatively new biomarker of aneurysm instability; therefore, there is no consensus about the best approach in quantifying aneurysm enhancement. Some studies have used the mean versus maximal SI. ${ }^{13,15,16,27}$ Saccular aneurysms with focal enhancement will have a lower mean SI as the ROI values are averaged and dilute focal enhancement. Even aneurysms with CAWE will have a higher mean SI. These 
variations may reflect an array of different morphological environments within the aneurysm wall and different stages of inflammation and/or vasculopathy. ${ }^{7}$ High SI values on areas of focal enhancement are associated with important structural changes of the aneurysm wall, including blebs, daughter sacs, microbleeds, intraluminal thrombus, neovascularization, and inflammatory cell infiltration. ${ }^{13,20,22}$ Such focal abnormalities in the aneurysm wall, although recorded as lower enhancement on HR-VWI when using mean SI values, are areas of instability that increase the risk of rupture. In this study, all enhancement ratios/indexes using maximal SI values achieved higher AUCs than those using mean SI values. We favor using the maximal SI regardless of the enhancement pattern (focal vs CAWE) when calculating enhancement ratios or indexes.

Clinical use of HR-VWI as a surrogate of aneurysm instability has been widely criticized for the lack of consensus regarding imaging protocols, heterogeneity in the definition of wall enhancement, and inability to reproduce the same results. ${ }^{11,24}$ Our study is the first to test the same HR-VWI protocol between scanners from different manufacturers and different potencies. Although absolute SI values measured on $3 \mathrm{~T}$ GE images were significantly lower than those measured on 3T Siemens images, the intermanufacturer correlation for $\mathrm{CR}_{\text {stalk }}$ using mean and maximal SI was almost perfect. On the other hand, AER and AEI results were not reproducible using different MRI scanners from both manufacturers. These findings reflect an important consideration for planning a multicenter prospective study of HR-VWI for characterization of UIAs.

MRI studies assessing SI of the normal pituitary stalk are well described in the literature. ${ }^{23}$ Contrast enhancement visualized in the pituitary stalk is attributed to the presence of hypothalamic axons directed to the neurohypophysis and varies depending on the thickness of their myelin sheath. ${ }^{21}$ In healthy individuals, the level of enhancement of the pituitary stalk does not seem to change significantly over the years, whereas the pineal gland, habenula, and ventricular choroid plexuses experience progressive physiological age-related calcification. ${ }^{1,29}$ The presence of calcium deposits in these structures affects their SI measurements and precludes their use as a reliable reference of enhancement in HR-VWI.

Moreover, the AEI measured in our study (normalized to the genu of the corpus callosum) could not be reproduced between MRI scanners from different manufacturers. The corpus callosum and other gray/white matter structures are contained inside the blood-brain barrier, and it is unlikely that they will show significant postcontrast enhancement. On the other hand, the pituitary stalk largely resides outside the blood-brain barrier and exhibits stable gadolinium enhancement. ${ }^{29}$ Therefore, the pituitary stalk may be the most appropriate intracranial structure to use as a reference when defining aneurysm wall enhancement on postcontrast MRI. In this study of 102 UIAs, the pituitary stalk was the most reliable and reproducible reference for normalization of SI in the analysis of aneurysm wall enhancement.

\section{Future Directions}

A previous trial that randomized UIAs to coiling versus conservative management failed due to slow recruitment. ${ }^{18}$ One of the major drawbacks of this trial was the lack of a biomarker of aneurysm instability. Patients and physicians faced the dichotomy of intervention versus observation. A new trial will have to include HR-VWI as a biomarker of aneurysm instability and may incorporate the following parameters: SI, quantitative susceptibility mapping, ${ }^{14}$ wall thickness, and advanced volumetric measurements. Patients and physicians will have to commit to participating in the study and follow through with randomization, even if it involves serial imaging without intervention. Unfortunately, there are no prospective clinical data to determine if a UIA with a $\mathrm{CR}_{\text {stalk }} \geq 0.60$ should be treated. Patients with 3- to 7-mm UIAs may undergo HR-VWI at diagnosis and follow-up. Aneurysm growth, morphological changes, development of new symptoms, or progression to rupture could be prospectively compared with parameters of aneurysm instability on HR-VWI.

\section{Limitations}

Our relatively small sample size limits the generalizability of the results. HR-VWI may be prone to more artifacts when analyzing smaller aneurysms located in the cavernous and paraclinoid segments of the internal carotid artery. The cavernous/sphenoid sinuses and dural folds of the skull base enhance with contrast and may be confounded with the aneurysm wall. In this study, 6 (5.9\%) aneurysms were in the cavernous portion of the internal carotid artery.

Additionally, our AUC-ROC curve analyses assumed that UIAs $\geq 7 \mathrm{~mm}$ located in the ACoA, PCoA, and BA were unstable. Although such an assumption is widely supported by the ISUIA ${ }^{27}$ and the UCAS (Unruptured Cerebral Aneurysm Study), ${ }^{9}$ the gold standard would be to evaluate aneurysm instability prospectively. The PHASES (population, hypertension, age, size of aneurysm, earlier SAH from another aneurysm, site of aneurysm) score was not used to define aneurysm instability due to the known limitations of this scale: underrepresentation of patients with familial aneurysms and young smokers, limited longterm follow-up data, and exclusion of the risk of intervention or treatment. ${ }^{6}$ Moreover, the PHASES score did not account for aneurysm morphology as a risk factor of rupture. ${ }^{17}$ No UIAs ruptured during follow-up in our cohort. A total of 12 patients with 16 UIAs underwent follow-up HR-VWI (mean 7.2 months). These patients will be followed prospectively to determine if there are changes in the degree of aneurysm wall enhancement and whether the UIAs grow or become symptomatic.

Another limitation when comparing SI across different scanners is the effect of manufacturer-specific sequences and techniques for fat suppression and image acquisition, and the associated hardware differences between scanners (32-channel head coil for the GE scanner vs 20-channel coil for the Siemens scanner). Nevertheless, these differences are more reflective of a real-world setting and likely to be encountered as HR-VWI is more frequently utilized.

\section{Conclusions}

$\mathrm{CR}_{\text {stalk }}$ using maximal SI values was the most reliable 
objective method to quantify aneurysm enhancement on HR-VWI. Aneurysms $\geq 7 \mathrm{~mm}$ located in the ACoA, PCoA, and BA showed increased wall enhancement. Adjusting aneurysm enhancement for SI values measured in the pituitary stalk allows standardization and reproducibility of results between MRI studies with scanners from different manufacturers and achieves higher sensitivity and specificity. These findings may help with standardization of quantifiable parameters of aneurysm enhancement and comparison of results across different manufacturer platforms.

\section{Acknowledgments}

This work was funded by the Brain Aneurysm Research grant from The Bee Foundation to Dr. Samaniego and conducted on an MRI instrument funded by NIH grant no. 1S10RR028821-01.

\section{References}

1. Araki Y, Ashikaga R, Takahashi S, Ueda J, Ishida O: High signal intensity of the infundibular stalk on fluid-attenuated inversion recovery MR. AJNR Am J Neuroradiol 18:89-93, 1997

2. Backes D, Hendrikse J, van der Schaaf I, Algra A, Lindgren AE, Verweij BH, et al: Determinants of gadoliniumenhancement of the aneurysm wall in unruptured intracranial aneurysms. Neurosurgery 83:719-725, 2018

3. Brinjikji W, Zhu YQ, Lanzino G, Cloft HJ, Murad MH, Wang $\mathrm{Z}$, et al: Risk factors for growth of intracranial aneurysms: a systematic review and meta-analysis. AJNR Am J Neuroradiol 37:615-620, 2016

4. Chalouhi N, Hoh BL, Hasan D: Review of cerebral aneurysm formation, growth, and rupture. Stroke 44:3613-3622, 2013

5. Edjlali M, Gentric JC, Régent-Rodriguez C, Trystram D, Hassen WB, Lion S, et al: Does aneurysmal wall enhancement on vessel wall MRI help to distinguish stable from unstable intracranial aneurysms? Stroke 45:3704-3706, 2014

6. Etminan N, Brown RD Jr, Beseoglu K, Juvela S, Raymond J, Morita A, et al: The unruptured intracranial aneurysm treatment score: a multidisciplinary consensus. Neurology 85:881-889, 2015

7. Frösen J, Cebral J, Robertson AM, Aoki T: Flow-induced, inflammation-mediated arterial wall remodeling in the formation and progression of intracranial aneurysms. Neurosurg Focus 47(1):E21, 2019

8. Hu P, Yang Q, Wang DD, Guan SC, Zhang HQ: Wall enhancement on high-resolution magnetic resonance imaging may predict an unsteady state of an intracranial saccular aneurysm. Neuroradiology 58:979-985, 2016

9. Hudson JS, Zanaty M, Nakagawa D, Kung DK, Jabbour P, Samaniego EA, et al: Magnetic resonance vessel wall imaging in human intracranial aneurysms. Stroke 50:e1, 2019

10. Larsen N, von der Brelie C, Trick D, Riedel CH, Lindner T, Madjidyar J, et al: Vessel wall enhancement in unruptured intracranial aneurysms: an indicator for higher risk of rupture? High-resolution MR imaging and correlated histologic findings. AJNR Am J Neuroradiol 39:1617-1621, 2018

11. Lehman VT, Brinjikji W: Vessel wall imaging of unruptured intracranial aneurysms: ready for prime time? Not so fast! AJNR Am J Neuroradiol 40:E26-E29, 2019

12. Lv N, Karmonik C, Chen S, Wang X, Fang Y, Huang Q, et al: Relationship between aneurysm wall enhancement in vessel wall magnetic resonance imaging and rupture risk of unruptured intracranial aneurysms. Neurosurgery 84:E385E391, 2019
13. Matsushige T, Shimonaga K, Mizoue T, Hosogai M, Hashimoto Y, Kaneko M, et al: Focal aneurysm wall enhancement on magnetic resonance imaging indicates intraluminal thrombus and the rupture point. World Neurosurg 127:e578-e584, 2019

14. Nakagawa D, Kudo K, Awe O, Zanaty M, Nagahama Y, Cushing C, et al: Detection of microbleeds associated with sentinel headache using MRI quantitative susceptibility mapping: pilot study. J Neurosurg 130:1391-1397, 2019

15. Omodaka S, Endo H, Niizuma K, Fujimura M, Inoue T, Endo T, et al: Circumferential wall enhancement in evolving intracranial aneurysms on magnetic resonance vessel wall imaging. J Neurosurg 131:1262-1268, 2019

16. Omodaka S, Endo H, Niizuma K, Fujimura M, Inoue T, Sato K, et al: Quantitative assessment of circumferential enhancement along the wall of cerebral aneurysms using MR imaging. AJNR Am J Neuroradiol 37:1262-1266, 2016

17. Ravindra VM, de Havenon A, Gooldy TC, Scoville J, Guan J, Couldwell WT, et al: Validation of the unruptured intracranial aneurysm treatment score: comparison with realworld cerebrovascular practice. J Neurosurg 129:100-106, 2018

18. Raymond J, Darsaut TE, Molyneux AJ: A trial on unruptured intracranial aneurysms (the TEAM trial): results, lessons from a failure and the necessity for clinical care trials. Trials 12:64, 2011

19. Samaniego EA, Roa JA, Hasan D: Vessel wall imaging in intracranial aneurysms. J Neurointerv Surg 11:1105-1112, 2019

20. Sato T, Matsushige T, Chen B, Gembruch O, Dammann P, Jabbarli R, et al: Wall contrast enhancement of thrombosed intracranial aneurysms at 7T MRI. AJNR Am J Neuroradiol 40:1106-1111, 2019

21. Satogami N, Miki Y, Koyama T, Kataoka M, Togashi K: Normal pituitary stalk: high-resolution MR imaging at 3T. AJNR Am J Neuroradiol 31:355-359, 2010

22. Shimonaga K, Matsushige T, Ishii D, Sakamoto S, Hosogai M, Kawasumi T, et al: Clinicopathological insights from vessel wall imaging of unruptured intracranial aneurysms. Stroke 49:2516-2519, 2018

23. Simmons GE, Suchnicki JE, Rak KM, Damiano TR: MR imaging of the pituitary stalk: size, shape, and enhancement pattern. AJR Am J Roentgenol 159:375-377, 1992

24. Texakalidis P, Hilditch CA, Lehman V, Lanzino G, Pereira VM, Brinjikji W: Vessel wall imaging of intracranial aneurysms: systematic review and meta-analysis. World Neurosurg 117:453-458.e1, 2018

25. Wadghiri YZ, Hoang DM, Leporati A, Gounis MJ, Rodríguez-Rodríguez A, Mazzanti ML, et al: High-resolution imaging of myeloperoxidase activity sensors in human cerebrovascular disease. Sci Rep 8:7687, 2018

26. Wang GX, Gong MF, Zhang D, Lei S, Yin JB, Gong ZL, et al: Wall enhancement ratio determined by vessel wall MRI associated with symptomatic intracranial aneurysms. Eur J Radiol 112:88-92, 2019

27. Wang GX, Wen L, Lei S, Ran Q, Yin JB, Gong ZL, et al: Wall enhancement ratio and partial wall enhancement on MRI associated with the rupture of intracranial aneurysms. J Neurointerv Surg 10:566-570, 2018

28. Wang X, Zhu C, Leng Y, Degnan AJ, Lu J: Intracranial aneurysm wall enhancement associated with aneurysm rupture: a systematic review and meta-analysis. Acad Radiol 26:664-673, 2019

29. Whitehead MT, Oh C, Raju A, Choudhri AF: Physiologic pineal region, choroid plexus, and dural calcifications in the first decade of life. AJNR Am J Neuroradiol 36:575-580, 2015

30. Wiebers DO, Whisnant JP, Huston J III, Meissner I, Brown RD Jr, Piepgras DG, et al: Unruptured intracranial 
aneurysms: natural history, clinical outcome, and risks of surgical and endovascular treatment. Lancet 362:103-110, 2003

\section{Disclosures}

Dr. Bathla: support of non-study-related clinical or research effort from Siemens Healthineers.

\section{Author Contributions}

Conception and design: Samaniego, Roa, Hasan. Acquisition of data: Roa, Osorno-Cruz. Analysis and interpretation of data: Roa, Bathla, Hasan. Drafting the article: Samaniego, Roa, Zanaty, Ishii, Bathla, Ortega-Gutierrez, Hasan. Critically revising the article: Samaniego, Roa, Hasan. Reviewed submitted version of manu- script: Samaniego, Roa. Approved the final version of the manuscript on behalf of all authors: Samaniego. Statistical analysis: Roa. Study supervision: Samaniego.

\section{Supplemental Information}

Online-Only Content

Supplemental material is available with the online version of the article.

Supplemental Tables 1-4. https://thejns.org/doi/suppl/10.3171/ 2019.12.JNS192746.

\section{Correspondence}

Edgar A. Samaniego: University of Iowa Hospitals and Clinics, Iowa City, IA. edgarsama@gmail.com. 